\title{
Simplified approach for quantitative digital holographic phase contrast imaging of living cells
}

\author{
Björn Kemper, ${ }^{a}$ Angelika Vollmer, ${ }^{a}$ Christina E. Rommel, ${ }^{b}$ Jürgen Schnekenburger, ${ }^{b}$ and Gert von Bally ${ }^{a}$ \\ a University of Muenster, Center for Biomedical Optics and Photonics, Robert-Koch-Str. 45, D-48149 Muenster, \\ Germany \\ bUniversity of Muenster, Gastroenterological Molecular Cell Biology, Department of Medicine B, D-48149 Muenster, \\ Germany
}

\begin{abstract}
Many interferometry-based quantitative phase contrast imaging techniques require a separately generated coherent reference wave. This results in a low phase stability and the demand for a precise adjustment of the intensity ratio between object and reference wave. To overcome these problems, the performance of a Michelson interferometer approach for digital holographic microscopy was analyzed that avoids a separately generated reference wave by superposition of different image areas. It is shown that this simplified arrangement yields improved phase stability. Furthermore, results from time-lapse investigations on living pancreas tumor cells demonstrate the capability of the method for reliable quantitative phase contrast imaging. @ 2011 Society of Photo-Optical Instrumentation Engineers (SPIE). [DOI: 10.1117/1.3540674]
\end{abstract}

Keywords: quantitative phase contrast; digital holographic microscopy; live cell imaging.

Paper 10538R received Oct. 1, 2010; revised manuscript received Nov. 25, 2010; accepted for publication Dec. 22, 2010; published online Feb. 10, 2011.

\section{Introduction}

Interferometry-based quantitative phase contrast techniques enable high-resolution topography inspection of reflective surfaces, ${ }^{1}$ label-free minimally invasive live cell analysis,${ }^{2-9}$ and quantitative tomographic imaging. ${ }^{10-14}$ However, a drawback of many experimental arrangements ${ }^{1,2,4-7,9-13}$ is the requirement for a separate reference wave, which results in a phase stability decrease and the demand for a precise adjustment of the intensity ratio between object and reference wave. To overcome these problems several approaches were reported. For example, in Fourier phase microscopy ${ }^{3}$ a programmable phase modulator is used for temporal phase shifting of a reference wave that is generated by the spatially filtered object wave. In diffraction phase microscopy, ${ }^{8}$ object and reference waves are generated by a diffractive optical element (DOE) in combination with a spatial filter. In spatial light phase microscopy, ${ }^{15}$ a spatial light modulator (SLM) is used to create a similar but more flexible setup. In quadriwave lateral shearing interferometry ${ }^{16}$ by a DOE, two orthogonal phase gradient maps are created from which quantitative phase contrast images are retrieved numerically. An interferometer setup in which the image of a sample is superimposed by an inverted image that is created by an additional lens in combination with movable mirrors to achieve an enhanced depth of field was reported in Ref. 17. Furthermore, in Ref. 18 an approach is described in which the reflective surface of a cover glass above the sample was used to generate a reference wave.

Here, in order to avoid a separately generated reference wave, a Michelson interferometer approach for digital holographic microscopy (DHM) is presented. The advantage of the proposed setup as compared to other self-interference or common

Address all correspondence to: Björn Kemper, University of Muenster, Center for Biomedical Optics and Photonics, Robert-Koch-Str. 45, D-48149 Muenster, Germany. E-mail: bkemper@uni-muenster.de. path-based approaches no additional components such as temporal phase shifting devices, ${ }^{3}$ lenses, ${ }^{17}$ or customized reflective surfaces ${ }^{18}$ are required. Furthermore, no optical elements such as $\mathrm{DOEs}^{16}$ or SLMs ${ }^{14,15}$ are used, which may be expensive or possibly affect the object wave by spatial filtering and thus decrease the lateral resolution.

\section{Experimental Setup and Hologram Evaluation}

Figure 1 shows a sketch and a photo of the experimental setup, which was attached to an inverted research microscope (Zeiss Axio Observer A1, Carl Zeiss Micro Imaging GmbH, Göttingen, Germany). The sample was illuminated in transmission with coherent light [frequency doubled Nd:yttrium aluminium garnet (YAG) laser: (Compass 100, Coherent GmbH, Lübeck, Germany), $\lambda=532 \mathrm{~nm}$ ] via a single mode optical fiber (SM). This was performed by inserting a nonpolarizing beam splitter cube (BS1) into the illumination path of the microscope's white light source (WS). Thus, the microscope condenser (CL) could be used for optimized (Koehler like) illumination. The sample (S) was imaged by a microscope lens (MO). Behind the MO the light was coupled into a Michelson interferometer arrangement, consisting of two mirrors (M1, M2) and a beam splitter cube (BS2), which was attached to the camera side port of the microscope. To create a suitable spatial carrier fringe pattern for off-axis holography, mirror M2 was tilted by an angle $\alpha$ in such a way that an area of the sample that contained no object was superposed with the image of the specimen. Note that due to the Michelson interferometer design in areas without specimen two wave fronts with nearly identical curvatures are superimposed. This is even fulfilled for an imaging geometry with two slightly divergent waves that differs from the collimated arrangement, which is sketched in Fig. 1(a) to simplify the illustration of the proposed measurement principle. Thus, an almost ideal pattern

1083-3668/2011/16(2)/026014/4/\$25.00 (C) 2011 SPIE 

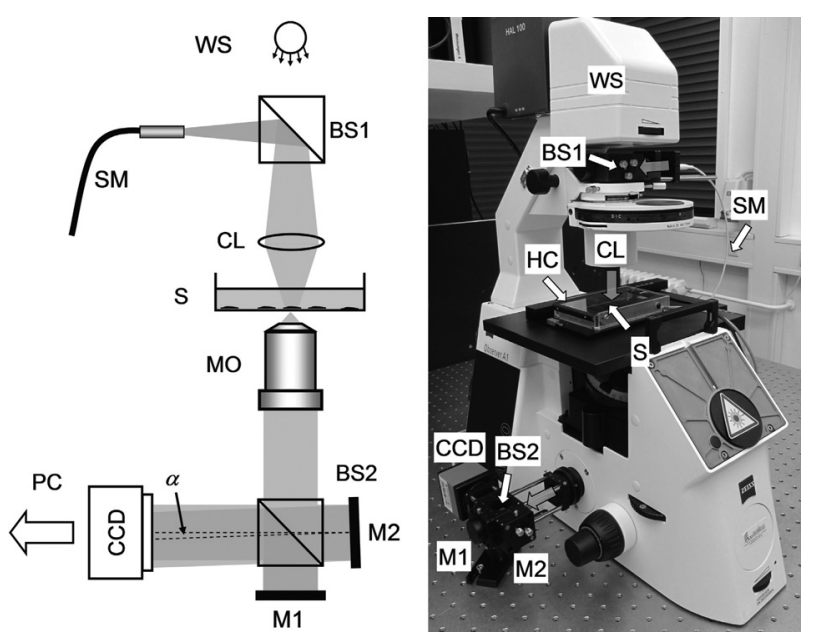

Fig. 1 Schematic (left) and photo (right) of the experimental setup. WS: white light source; BS1, BS2: beam splitter cubes; CL: condenser lens; SM: single mode fiber; S: sample (here: Petri dish with adherent cells); MO: microscope lens; M1, M2: mirrors; CCD: charge coupled device sensor; $\alpha$ : tilt angle; PC: computer; HC: heating chamber.

of parallel off-axis spatial carrier fringes is formed. In contrast to Mach-Zehnder setups, as for example described in Refs. $1,2,5-7,9-12$, and 13 , the fringe curvature and orientation as well as the spatial carrier frequency do not depend on the object illumination alignment. Furthermore, two waves with almost identical intensity and polarization are created. Hence, a maximized constant interference fringe contrast is achieved, which is independent of the polarization and the intensity of the object illumination. The resulting digital off-axis holograms were recorded by a charge coupled device sensor (CCD, The Imaging Source DMK 41BU02, Bremen, Germany) and transferred via a USB 2.0 interface to a computer. The numerical reconstruction of the quantitative DHM phase contrast images from the digital holograms was performed by spatial phase shifting-based reconstruction as reported previously in Refs. 2 and 7. In the case of unfocused imaging, numerical autofocusing was applied as described in Ref. 19.

\section{Cell Preparation}

In order to analyze the capability of the method for quantitative phase contrast imaging, investigations on living human pancreas tumor cells [PaTu8988T, (Ref. 7)] were performed. The cells were observed in Petri dishes ( $\mu$-Dish, ibidi GmbH, Munich, Germany) with a collagen I coated bottom in cell culture medium (DMEM, supplemented with 5\% fetal calf serum, 5\% horse serum, and 2-mM L-glutamine, buffered with 20-mM Hepes). A heating chamber (HT200, ibidi GmbH, Munich, Germany) was used for temperature stabilization at $37^{\circ} \mathrm{C}$.

\section{Results}

Figure 2 illustrates the measurement principle by white light images and the evaluation of a digital hologram that were obtained from three living PaTu8988T cells by using a $40 \times$ microscope lens (Zeiss LD Plan-Neofluar 40/0.6 Korr). For both, white light imaging and hologram recording, the specimens were sharply focused onto the CCD sensor. Figures2(a)-2(c) show white light
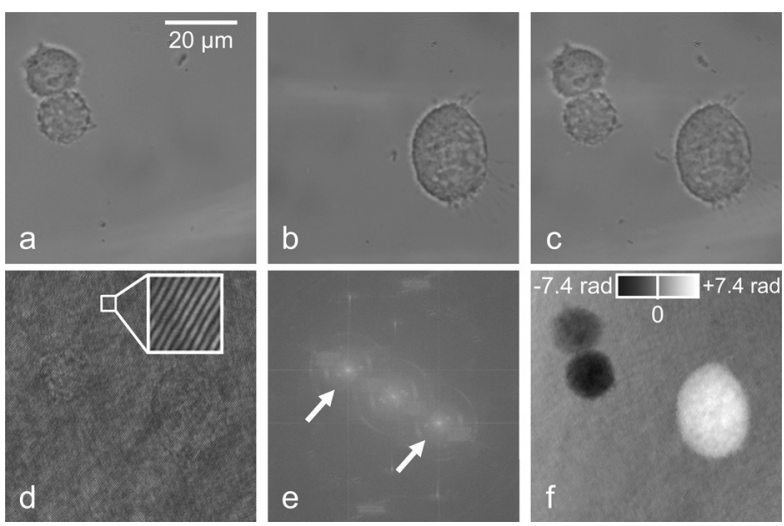

Fig. 2 Principles of white light imaging and hologram evaluation. (a) white light image of PaTu8988T cells from mirror M1 (M2 blocked); (b) white light image from mirror M2 (M1 blocked); (c) white light image from $\mathrm{M} 1+\mathrm{M} 2$; (d) digital off-axis hologram with enlarged spatial carrier fringe pattern; (e) two dimensional frequency spectrum of (d); (f) quantitative phase contrast image numerically reconstructed from (d) (coded to 256 gray levels).

images of the cells obtained from mirror M1 (optical path of mirror M2 blocked), from mirror M2 (optical path of mirror M1 blocked), and the superimposed image obtained by using both mirrors, M1 and M2. In Fig. 2(d) a digital hologram is depicted which corresponds to the configuration in Fig. 2(c). For hologram recording, the white light illumination was replaced by the coherent light of the frequency doubled Nd:YAG laser. The angle $\alpha$ between the two mirrors M1, M2 was chosen in such way that a spatial carrier fringe frequency with a spatial phase gradient near $0.5 \pi$ per pixel $^{20}$ for optimized numerical reconstruction was generated. The resulting parallel carrier fringe pattern is depicted in the enlarged part of Fig. 2(d). Figure 2(e) shows the spatial frequency spectrum of the hologram in Fig. 2(d) that was calculated by a two-dimensional fast Fourier transformation (FFT). The narrow peaks that are marked in Fig. 2(d) with arrows demonstrate the high linear degree of the carrier fringe pattern. Finally, Fig. 2(e) presents the reconstructed quantitative phase contrast image. Each of the two sheared wave fronts serves as a reference wave for the complementary wave. Thus, the cells appear in inverse phase contrast as they are imaged with different mirrors.

To characterize the phase stability of the Michelson interferometer approach, comparative time-lapse investigations with the setup sketched in Fig. 1 and a modular DHM system based on a Mach-Zehnder interferometer with a fiber optic reference wave (see detailed description in Ref. 21) were performed. The experiments were performed without a sample on the same vibration isolated table. Both systems were attached to the same Zeiss Axio Observer A1 microscope. Figures 3(a) and 3(b) exemplarily show the temporal dependency of the reconstructed phase distributions $\Delta \varphi(t)$ in an area of $5 \times 5$ pixels as well as the corresponding calculated optical path length (OPL) changes $\Delta \mathrm{OPL}=\lambda(\Delta \varphi / 2 \pi)$ for periods of 1 and $60 \mathrm{~min}$. The phase contrast images were reconstructed from digital holograms that were recorded with acquisition rates of 1 hologram per second for the period of 1 minute and 1 hologram per minute for the period of $60 \mathrm{~min}$. For the proposed Michelson interferometer setup an up to 5 times lower value for the standard deviations $\sigma_{\Delta \varphi, t}$ and $\sigma_{\Delta \mathrm{OPL}, t}$ of the measured $\Delta \varphi(t)$ and $\Delta \mathrm{OPL}(t)$ values than 

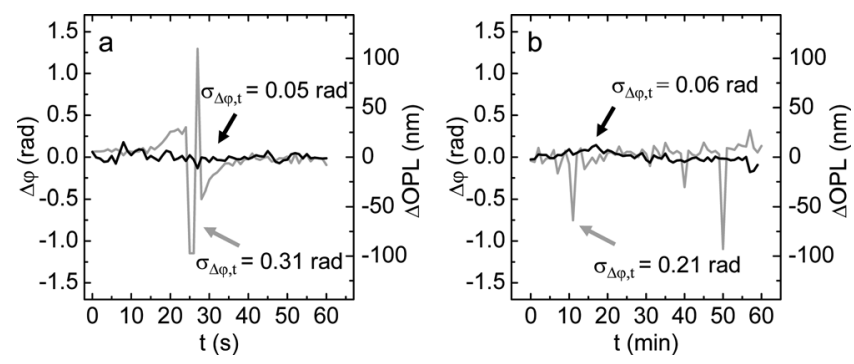

Fig. 3 Comparison of the temporal phase stability $\Delta \varphi(t)$ of the simplified DHM concept to a fiber optic-based Mach-Zehnder setup (a) for a period of 1 min (acquisition rate one hologram per second); the standard deviations of the temporal phase changes $\sigma_{\Delta \varphi}, t$ correspond to the mean accuracies for the detection of optical path length changes $\sigma_{\Delta \mathrm{OPL}, t}=4.2 \mathrm{~nm}$ (simplified DHM setup), $\sigma_{\Delta \mathrm{OPL}, t}=26.0 \mathrm{~nm}$ $=26.0 \mathrm{~nm}$ (Mach-Zehnder setup); and (b) for a period of $60 \mathrm{~min}$ (acquisition rate one hologram per min); the standard deviations of the temporal phase change $\sigma_{\Delta \varphi, t}$ correspond to the mean accuracies for the detection of optical path length changes $\sigma_{\Delta \mathrm{OPL}, t}=5.3 \mathrm{~nm}$ (simplified DHM setup), $\sigma_{\Delta O P L} t=17.7 \mathrm{~nm}$ (Mach-Zehnder setup); black line: simplified Michelson interferometer setup, green line: fiber optic-based Mach-Zehnder interferometer.

for the Mach-Zehnder interferometer-based setup is obtained. The peaks that are observed in Fig. 3 for $\Delta \varphi(t)$ and $\Delta \operatorname{OPL}(t)$ for the Mach-Zehnder setup may be explained by thermal drifts and air fluctuations. These disturbances induce phase difference changes between the two fiber optical paths which appear significantly reduced in the Michelson interferometer setup. In order to compare the accuracy for the detection of optical path length changes within single quantitative phase contrast images the phase noise $\sigma_{\Delta \varphi, x, y}$ was quantified for each system without a sample as described in Ref. 22. For the Michelson interferometer setup $\sigma_{\Delta \varphi, x, y}$ was determined to $0.16 \mathrm{rad}$ while for the Mach-Zehnder setup $\sigma_{\Delta \varphi, x, y}$ amounted to 0.09 rad. This corresponds to mean accuracies for the detection of optical path length changes $\sigma_{\triangle \mathrm{OPL}, x, y}$ of 13.6 and $7.6 \mathrm{~nm}$.

Finally, in time-lapse measurements the migration of the PaTu8988T cells was analyzed. Therefore, 128 digital off-axis holograms of selected cells were recorded every 3 min over a period of $6.3 \mathrm{~h}$ by using a $20 \times$ microscope lens (Zeiss LD

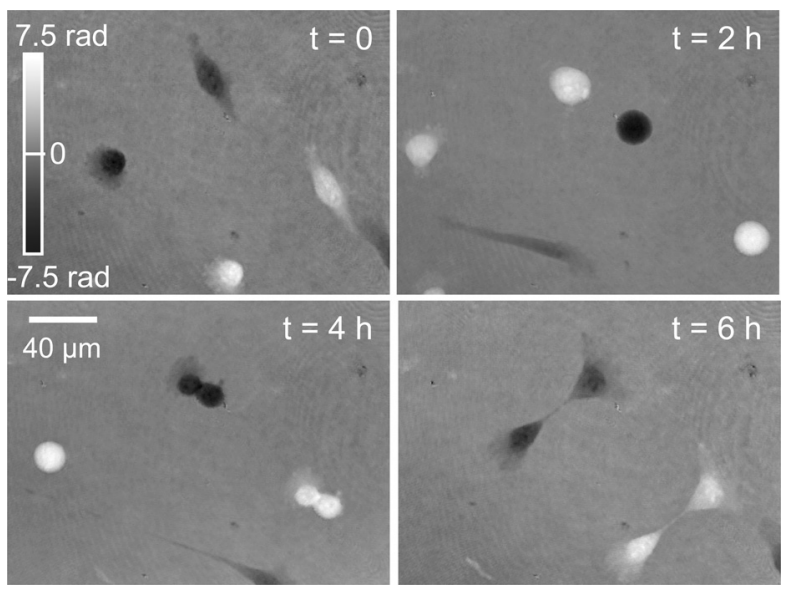

Fig. 4 Quantitative DHM phase contrast images of living PaTu8988T cells on a collagen coated surface for $t=0, t=2 \mathrm{~h}, t=4 \mathrm{~h}, t=$ $6 \mathrm{~h}$. Video 1 shows a corresponding fast motion movie. (QuickTime, 1.4MB) [URL: http://dx.doi.org/10.1117/1.3540674.1]
Acroplan $20 \times / 0.4$ Korr). Figure 4 shows exemplary quantitative phase contrast images. Video 1 (see Fig. 4) shows a corresponding fast motion movie. Subcellular structures like the nuclear envelope and the nucleoli as well as the thin borders of the cells become visible. Furthermore, cell migration and cell division can be clearly observed. Due to the high phase stability of the Michelson interferometer arrangement, $95 \%$ of the quantitative phase contrast images were obtained without reconstruction artifacts such as phase unwrapping errors. Note that cells that are imaged by different mirrors can be identified as nonambiguous as they appear in inverse phase contrast.

\section{Discussion and Conclusions}

In summary, a significantly simplified and cost efficient approach for DHM with very low alignment demands was presented, which is capable for quantitative phase contrast imaging of living cells. The arrangement can be attached as a modular add-on to common research microscopes. The measurement concept leads to improved temporal phase stability in comparison to fiber optic Mach-Zehnder interferometer-based DHM systems. It has to be mentioned that due to the specific design of the experimental setup, specimen free reference areas (SFRAs) near the sample are required. The SFRAs should be at least the size of the investigated sample (here: the lateral cell diameter which amounts to $\approx 30 \mu \mathrm{m}$ ). Thus, the method is currently limited to single cell analysis with low densities of adherent or suspended cells in which the specimen appear laterally separated within the field of view. However, this problem may be solved by observation chambers with adequately structured coated bottom areas that prevent cell adherence. The analysis of the spatial phase noise within the quantitative phase contrast images shows that the accuracy for the detection of optical path length changes for the simplified DHM arrangement is lower than for a MachZehnder setup. This may be explained by the circumstance that in the Michelson interferometer setup both waves are affected by coherent disturbances due to scattering effects from the microscope imaging system. Furthermore, for the described live cell imaging experiments, both waves pass the same volume of the chamber in which the cells are observed. Thus, additional scattering effects of the cell culture medium can be expected also in SFRAs. However, such disturbances individually depend on the applied measurement setup and the sample preparation. Nevertheless, Fig. 4 demonstrates that the proposed method is capable for label-free live cell imaging with subcellular resolution even for long-term migration observations. Thus, the simplified DHM concept prospects to be a versatile tool to generate quantitative phase contrast data in a very simple way, which may be further evaluated to quantify cell adherence and tumor cell motility, as well as for dry mass measurements and the analysis of the cellular refractive index. Moreover, due to almost identical optical path lengths within the interferometer, the system is also particularly suitable for the use of low cost light sources with a short coherence length. In addition, by using light sources with a decreased coherence length an improvement of the quantitative phase contrast images due to a minimization of disturbing coherence effects may be achieved. Finally, as the spatial carrier fringes are not affected by the object wave alignment, the setup prospects to be used in tomographic phase microscopy ${ }^{10-13}$ of transparent and reflective samples with suitable SFRAs. Here, 
a simplification of the experimental setup and an acceleration of the data acquisition time by application of spatial shifting based phase retrieval instead of temporal phase shifting may be achieved.

\section{Acknowledgments}

This work was partly supported by the German Federal Ministry for Education and Research within the focus program "Biophotonics" (FKZ13N10937).

\section{References}

1. E. Cuche, P. Marquet, and C. Depeursinge, "Simultaneous amplitude contrast and quantitative phase-contrast microscopy by numerical reconstruction of Fresnel off-axis holograms," Appl. Opt. 38, 6694-7001 (1999).

2. D. Carl, B. Kemper, G. Wernicke, and G. von Bally, "Parameter optimized digital holographic microscope for high-resolution living cell analysis," Appl. Opt. 43, 6536-6544 (2004).

3. G. Popescu, L. P. Deflores, J. C. Vaughan, K. Badizadegan, H. Iwai, R. R. Dasari, and M. S. Feld, "Fourier phase microscopy for investigation of biological structure and dynamics," Opt. Lett. 29, 2503-2505 (2004).

4. P. Marquet, B. Rappaz, P. J. Magistretti, E. Cuche, Y. Emery, T. Colomb, and C. Depeursinge, "Digital holographic microscopy: a noninvasive contrast imaging technique allowing quantitative visualization of living cells with subwavelength axial accuracy," Opt. Lett. 30, 468-470 (2005)

5. C. J. Mann, L. F. Yu, C. M. Lo, and M. K. Kim, "High-resolution quantitative phase-contrast microscopy by digital holography," Opt. Express 13, 8693-8698 (2005).

6. T. Ikeda, G. Popescu, R. R. Dasari, and M. S. Feld, "Hilbert phase microscopy for investigating fast dynamics in transparent systems," Opt. Lett. 30, 1165-1167 (2005).

7. B. Kemper, D. Carl, J. Schnekenburger, I. Bredebusch, M. Schäfer, W. Domschke, and G. von Bally, "Investigation of living pancreas tumor cells by digital holographic microscopy," J. Biomed. Opt. 11, 034005 (2006).

8. G. Popescu, T. Ikeda, R. R. Dasari, and M. S. Feld, "Diffraction phase microscopy for quantifying cell structure and dynamics," Opt. Lett., $\mathbf{3 1}$, 775-778 (2006).

9. G. Coppola, G. Di Caprio, M. Gioffré, R. Puglisi, D. Balduzzi, A. Galli, L. Miccio, M. Paturzo, S. Grilli, A. Finizio, and P. Ferraro, "Dig- ital self-referencing quantitative phase microscopy by wavefront folding in holographic image reconstruction," Opt. Lett. 35, 3390-3392 (2010).

10. V. Lauer, "New approach to optical diffraction tomography yielding a vector equation of diffraction tomography and a novel tomographic microscope," J. Microsc. 205, 165-176 (2002).

11. W. Choi, C. Fang-Yen, K. Badizadegan, S. Oh, N. Lue, R. R. Dasari, and M. S. Feld "Tomographic phase microscopy," Nature Meth. 4, 717-719 (2007).

12. M. Debailleul, V. Georges, B. Simon, R. Morin, and O. Haeberlé, "Highresolution three-dimensional tomographic diffractive microscopy of transparent inorganic and biological samples," Opt. Lett. 34, 79-81 (2009).

13. M. Sarmis, B. Simon, M. Debailleul, B. Colicchio, V. Georges, J.-J. Delaunay, and O. Haeberlé, "High resolution reflection tomographic diffractive microscopy," J. Mod. Opt. 57, 740-745 (2010).

14. R. Fiolka, K. Wicker, R. Heintzmann, and A. Stemmer, "Simplified approach to diffraction tomography in optical microscopy," Opt. Express 17, 12407-12417 (2009).

15. H. Ding and G. Popescu, "Instantaneous spatial light interference microscopy," Opt. Express 18, 1569-1575 (2010).

16. P. Bon, G. Maucort, B. Wattellier, and S. Monneret, "Quadriwave lateral shearing interferometry for quantitative phase microscopy of living cells," Opt. Express 17, 13080-13094 (2009).

17. J. Jang, C. Y. Bae, J.-K. Park, and J. C. Ye, "Self-reference quantitative phase microscopy for microfluidic devices," Opt. Lett. 35, 514-516 (2010).

18. N. T. Shaked, Y. Zhu, N. Badie, N. Bursac, and A. Wax, "Reflective interferometric chamber for quantitative phase imaging of biological sample dynamics," J. Biomed. Opt. 15030503 (2010).

19. P. Langehanenberg, B. Kemper, D. Dirksen, and G. von Bally, "Autofocusing in digital holographic phase contrast microscopy on pure phase objects for live cell imaging," Appl. Opt. 47, D176-D182 (2008).

20. B. Kemper, J. Kandulla, D. Dirksen, and G. von Bally, "Optimization of spatial phase shifting in endoscopic electronic-speckle-patterninterferometry," Opt. Commun. 217, 151-160 (2003).

21. B. Kemper, D. Carl, A. Höink, G. von Bally, I. Bredebusch, and J. Schnekenburger, "Modular digital holographic microscopy system for marker free quantitative phase contrast imaging of living cells," Proc. SPIE 6191, 61910T (2006).

22. C. Remmersmann, S. Stürwald, B. Kemper, P. Langehanenberg, and G. von Bally, "Phase noise optimization in temporal phase-shifting digital holography with partial coherence light sources and its application in quantitative cell imaging," Appl. Opt. 48, 1463-1472 (2009). 